\title{
Research on Vibration of Automobile Suspension Design
}

\author{
Jun Yang, Mingming Dong \\ School of mechanical and Vehicular Engineering, Beijing Institute of Technology, Beijing, 100081, China
}

\begin{abstract}
In this paper, a seven-degree-of-freedom three-dimensional vibration model of a vehicle is established based on the parameters of a vehicle. Considering the hysteresis effect of pavement incentives of rear wheel, the random vibration of the vehicle is simulated by MATLAB / SIMULINK. After changing the stiffness, the acceleration of the vehicle body and the seat, suspension dynamic deflection and relative dynamic load are compared to select the suitable stiffness to achieve optimal ride performance. It can provide a reference for the one who is designing vehicle suspension.
\end{abstract}

\section{Introduction}

When the automobile is running, the vibration of the car is excited by the uneven surface and the rotating parts such as the transmission system and the wheels. Typically, uneven pavement is the basic input of automotive vibration. Solving the vibration problem of vehicle and parts under the excitation of random uneven road is an important foundation to improve vehicle ride comfort, safety and reliability of parts [1]. In this paper, the 7-DOF dynamics model of the vehicle's vehicle vibration system will be established, and the parameters of suspension will be optimized by using the vehicle body acceleration root mean square value, suspension dynamic deflection and wheel displacement as the ride comfort evaluation index.

\section{Vehicle vibration mechanics model}

In order to more realistic reflection of the vertical vibration of the vehicle, it is necessary to establish a three-dimensional vehicle vibration model. The vibration input of the whole vehicle is the excitation of the road surface, the difference of road excitation between the front and rear wheels, and the influence of the roll of the vehicle on the vibration of the vehicle body are taken into account, which is a more realistic reflection of the actual situation of vehicle vibration[2]. Seven-DOF threedimensional vehicle vibration model is shown in Figure 1.

$m_{b}, J_{b}, I_{b}$ are respectively the body mass, pitching inertia and yawing inertia. $m_{i}, c_{i}, k_{i}(i=1,2,3,4)$ are respectively the mass, damping and stiffness of the suspension. $c_{t i}, k_{t i}(\mathrm{i}=1,2,3,4)$ are respectively damping and stiffness of the wheel; $b_{f}$ is half of front wheel track. $b_{r}$ is half of front wheel track. $a$ is the distance between body mass center and front suspension. $b$ is the distance between body mass center and rear suspension. $Z_{b}, \theta, \varphi$ are respectively vertical displacement of car body, rate of pitch and yaw velocity. $z_{b i}, z_{i}$ are respectively vertical displacement of four supporting points of the car body and vertical displacement of the tire. $q_{i}$ are four wheel input of pavement excitation.

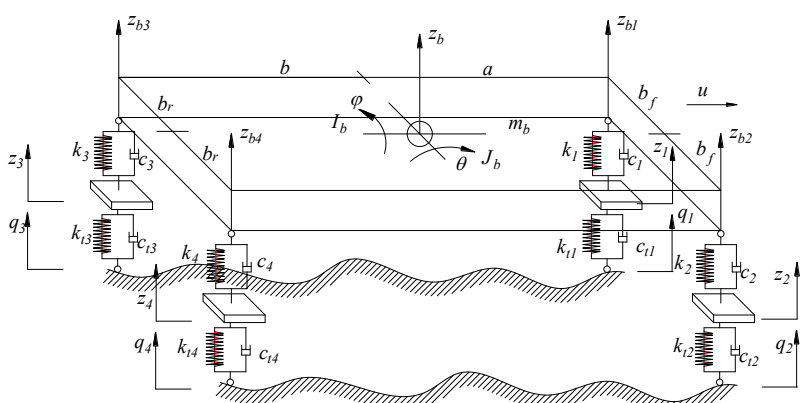

Figure 1. Vehicle vibration mechanics model.

According to the previous symbol definition,

$$
\left\{\begin{array}{l}
z_{b 1}=z_{b}-a \theta+b_{f} \varphi \\
z_{b 2}=z_{b}-a \theta-b_{f} \varphi \\
z_{b 3}=z_{b}+b \theta+b_{r} \varphi \\
z_{b 4}=z_{b}+b \theta-b_{r} \varphi
\end{array} .\right.
$$

The total kinetic energy of this system is:

$$
T=\frac{1}{2}\left[m_{b} \dot{z}_{b}^{2}+J_{b} \dot{\theta}^{2}+m_{1} \dot{z}_{1}^{2}+m_{2} \dot{z}_{2}^{2}+m_{3} \dot{z}_{3}^{2}+m_{4} \dot{z}_{4}^{2}\right] .
$$

The potential energy of this system is:

$$
U=\frac{1}{2}\left[\sum_{i=1}^{4} k_{i}\left(z_{b i}-z_{i}\right)^{2}+\sum_{i=1}^{4} k_{t i}\left(z_{i}-q_{i}\right)^{2}\right] .
$$

The total dissipation function of the system is: 


$$
R=\frac{1}{2}\left[\sum_{i=1}^{4} c_{i}\left(\dot{z}_{b i}-\dot{z}_{i}\right)^{2}+\sum_{i=1}^{4} c_{t i}\left(\dot{z}_{i}-\dot{q}_{i}\right)^{2}\right]
$$

After the above equations are arranged, the matrix form is obtained according to Lagrange's law:

$$
M \ddot{Z}+C \dot{Z}+K Z=K_{t} Q+C_{t} \dot{Q} .
$$

where $k_{1}=k_{2}, k_{3}=k_{4}, c_{1}=c_{2}, c_{3}=c_{4}$,

$$
\begin{aligned}
& M=\operatorname{diag}\left\{m_{b} \quad J_{b} \quad I_{b} \quad m_{1} \quad m_{2} \quad m_{3} \quad m_{4}\right\}, \\
& K=\left[\begin{array}{ccccccc}
2\left(k_{1}+k_{3}\right) & 2\left(b k_{3}-a k_{1}\right) & 0 & -k_{1} & -k_{1} & -k_{3} & -k_{3} \\
2\left(b k_{3}-a k_{1}\right) & 2\left(a^{2} k_{1}+b^{2} k_{3}\right) & 0 & a k_{1} & a k_{1} & -b k_{3} & -b k_{3} \\
0 & 0 & 2\left(b_{f}{ }^{2} k_{1}+b_{r}{ }^{2} k_{3}\right) & -b_{f} k_{1} & b_{r} k_{3} & -b_{f} k_{1} & b_{r} k_{3} \\
-k_{1} & a k_{1} & -b_{f} k_{1} & k_{1}+k_{t 1} & 0 & 0 & 0 \\
-k_{1} & a k_{1} & b_{f} k_{1} & 0 & k_{1}+k_{t 1} & 0 & 0 \\
-k_{3} & -b k_{3} & -b_{r} k_{3} & 0 & 0 & k_{3}+k_{t 3} & 0 \\
-k_{3} & -b k_{3} & b_{r} k_{3} & 0 & 0 & 0 & k_{3}+k_{t 3}
\end{array}\right], \\
& C=\left[\begin{array}{ccccccc}
2\left(c_{1}+c_{3}\right) & 2\left(b c_{3}-a c_{1}\right) & 0 & -c_{1} & -c_{1} & -c_{3} & -c_{3} \\
2\left(b c_{3}-a c_{1}\right) & 2\left(a^{2} c_{1}+b^{2} c_{3}\right) & 0 & a c_{1} & a c_{1} & -b c_{3} & -b c_{3} \\
0 & 0 & 2\left(b_{f}{ }^{2} c_{1}+b_{r}{ }^{2} c_{3}\right) & -b_{f} c_{1} & b_{r} c_{3} & -b_{f} c_{1} & b_{r} c_{3} \\
-c_{1} & a c_{1} & -b_{f} c_{1} & c_{1}+c_{t 1} & 0 & 0 & 0 \\
-c_{1} & a c_{1} & b_{f} c_{1} & 0 & c_{1}+c_{t 1} & 0 & 0 \\
-c_{3} & -b c_{3} & -b_{r} c_{3} & 0 & 0 & c_{3}+c_{t 3} & 0 \\
-c_{3} & -b c_{3} & b_{r} c_{3} & 0 & 0 & 0 & c_{3}+c_{t 3}
\end{array}\right] \\
& C_{t}=\left[\begin{array}{cccc}
0 & 0 & 0 & 0 \\
0 & 0 & 0 & 0 \\
0 & 0 & 0 & 0 \\
c_{t 1} & 0 & 0 & 0 \\
0 & c_{t 2} & 0 & 0 \\
0 & 0 & c_{t 3} & 0 \\
0 & 0 & 0 & c_{t 4}
\end{array}\right], \quad K_{t}=\left[\begin{array}{cccc}
0 & 0 & 0 & 0 \\
0 & 0 & 0 & 0 \\
0 & 0 & 0 & 0 \\
k_{t 1} & 0 & 0 & 0 \\
0 & k_{t 2} & 0 & 0 \\
0 & 0 & k_{t 3} & 0 \\
0 & 0 & 0 & k_{t 4}
\end{array}\right], \quad Z=\left[\begin{array}{c}
z_{b} \\
\theta \\
\varphi \\
z_{1} \\
z_{2} \\
z_{3} \\
z_{4}
\end{array}\right], \quad Q_{t}=\left[\begin{array}{c}
q_{1} \\
q_{2} \\
q_{3} \\
q_{4}
\end{array}\right] .
\end{aligned}
$$

\section{Simulation optimization}

\subsection{The establishment of pavement simulation model}

In the process of vehicle running, the roughness of the road surface varies with different grades of road and different driving speed. The pavement model is a mathematical description of the change of the road roughness with the class and speed of the road. The Seven Free 3D model of vehicle is built, which considers the rear wheel lag road roughness input, without considering the left and right coherent road input.

The pavement excitation is generated by the white noise method[3-6] and the mathematical model of pavement excitation is.

$$
\dot{q}(t)=-2 \pi n_{l} u q(t)+2 \pi n_{0} \sqrt{G_{q}\left(n_{0}\right) u} w(t),
$$

where $q(t)$ is four wheel input of pavement excitation; $w(t)$ is white noise signal which the mean is zero; $n_{l}$ is lower cut-off frequency, the value of which is $0.011 \mathrm{~m}^{-1} ; u$ is speed of vehicle, the value of which is $110 \mathrm{~km} / \mathrm{h} ; G_{q}\left(n_{0}\right)$ is the road power spectral density value under the reference road frequency, which is called the unevenness parameters of road surface. The road grade is usually divided according to the unevenness parameters of road surface.
According to Equation (10), the MATLAB / SIMULINK model is as follows:

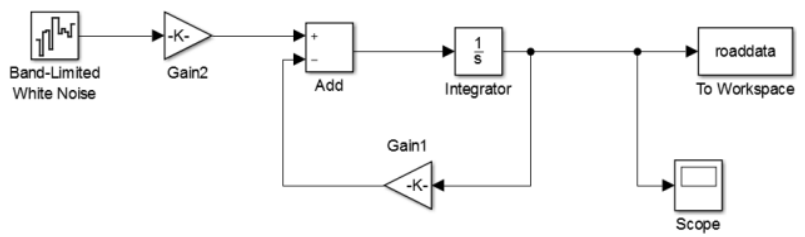

Figure 2. Pavement simulation model.

where Gain1 $=-2 \pi n_{l} u$, Gain $2=2 \pi n_{0} \sqrt{G_{q}\left(n_{0}\right) u}, n_{0}=$ $0.1 \mathrm{~m}^{-1}, G_{q}\left(n_{0}\right)=16 \times 10^{-5} \mathrm{~m}^{2} / \mathrm{m}^{-1}, \quad u=100 \mathrm{~km} / \mathrm{h}$.

The rear wheel road irregularity excitation delay for a period of time compared to the front [7]. The delay time $\tau$ is:

$$
\tau=\frac{a+b}{u} .
$$

\subsection{The establishment of vehicle simulation model}

In the second chapter, the three-dimensional vibration model of seven-degree-of-freedom vehicle was established and the differential equation of vehicle vibration was obtained. Ignoring tire damping, it is:

$$
M \ddot{Z}+C \dot{Z}+K Z=K_{t} Q .
$$

In order to facilitate the calculation and simulation, it is necessary to convert equation (12) into the form of state equation by multiplied $M^{-1}$. After transformation, the equation is:

$$
\ddot{Z}=-M^{-1} C \dot{Z}-M^{-1} K Z+M^{-1} K_{t} Q .
$$

The seven - freedom vehicle vibration simulation model is built by using the MATLAB/SIMULIK module, as shown in Figure 3.

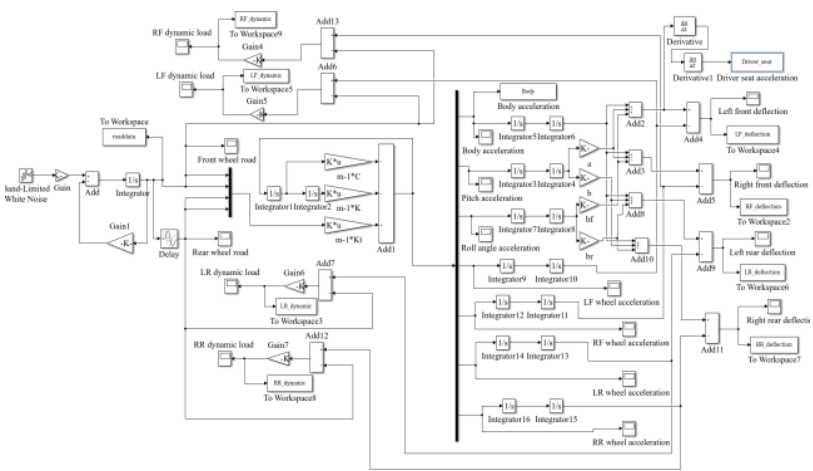

Figure 3. Vehicle simulation model

\subsection{Optimization}

The objective function is $\min (\ddot{z})$, which is to meet the requirements of ride comfort. Design variable is :

$$
X=\left[k_{1}, k_{3}\right]^{\mathrm{T}} .
$$


Static deflection of front and rear suspension $f_{e}$ is bigger than $0.10 \mathrm{~m}$ and lower than $0.30 \mathrm{~m}$. So the stiffness limit of the front and rear suspension is

$$
m_{1} g / 0.30 \leq k_{1} \leq m_{1} g / 0.10, m_{3} g / 0.30 \leq k_{3} \leq m_{3} g / 0.10,
$$

where $m_{1}, m_{3}$ are respectively left front and left rear suspension sprung mass. In order to improve the comfort of the driver, the frequency of the front suspension is often lower than that of the rear suspension. There is :

$$
k_{1} / k_{3}<m_{1} / m_{3},
$$

The parameters of a car are shown in Table 1.

\begin{tabular}{|c|c|c|c|}
\hline $\begin{array}{l}m_{b} / \\
\mathrm{kg}\end{array}$ & $\begin{array}{l}m_{1} / \\
\text { kg }\end{array}$ & $\begin{array}{l}m_{3} / \\
\text { kg }\end{array}$ & $\begin{array}{c}J_{b} / \\
\left(\mathbf{k g} \cdot \mathbf{m}^{2}\right)\end{array}$ \\
\hline 1380 & 40.5 & 45.4 & 2440 \\
\hline $\begin{array}{c}I_{b} \\
/\left(\mathbf{k g} \cdot \mathbf{m}^{2}\right)\end{array}$ & $\begin{array}{c}k_{1} / \\
(\mathrm{kN} / \mathrm{m})\end{array}$ & $\begin{array}{c}k_{3} / \\
(k N / m)\end{array}$ & $\begin{array}{c}c_{1} / \\
\left(\mathrm{kN} / \mathrm{s} \cdot \mathrm{m}^{-1}\right)\end{array}$ \\
\hline 80 & 17 & 22 & 1.5 \\
\hline $\begin{array}{c}c_{3} / \\
\left(\mathrm{kN} / \mathrm{s} \cdot \mathrm{m}^{-1}\right)\end{array}$ & $\begin{array}{c}k_{t 1} / \\
(\mathbf{k N} / \mathbf{m})\end{array}$ & $\begin{array}{c}k_{t 2} / \\
(\mathbf{k N} / \mathbf{m})\end{array}$ & $\begin{array}{l}a / \\
\mathbf{m}\end{array}$ \\
\hline 15 & 40.5 & 45.4 & 1.51 \\
\hline $\begin{array}{l}b / \\
m\end{array}$ & $\begin{array}{l}b_{r} / \\
m\end{array}$ & $\begin{array}{c}\boldsymbol{b}_{\boldsymbol{f}} / \\
\mathrm{m}\end{array}$ & \\
\hline 1.51 & 0.74 & 0.74 & \\
\hline
\end{tabular}

Table 1. Parameters of a car

According to the above requirements, the vehicle model is simulated and the simulation results by using improved and unimproved data are compared[7-8]. The simulation time is $30 \mathrm{~s}$. To see the details, let's take $5 \sim 10 \mathrm{~s}$ as a contrast.

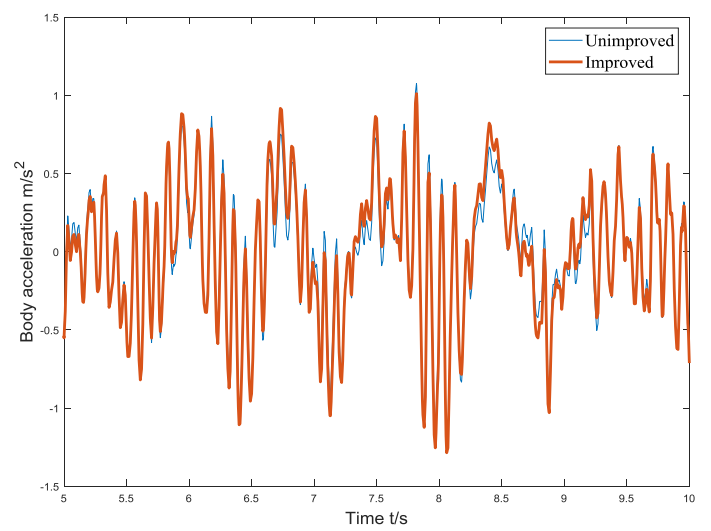

Figure 4. Comparison of body acceleration.

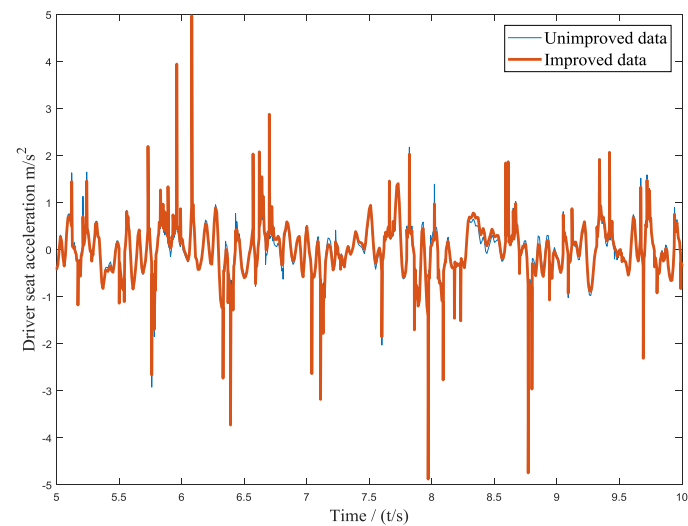

Figure 5. Comparison of driver seat acceleration.

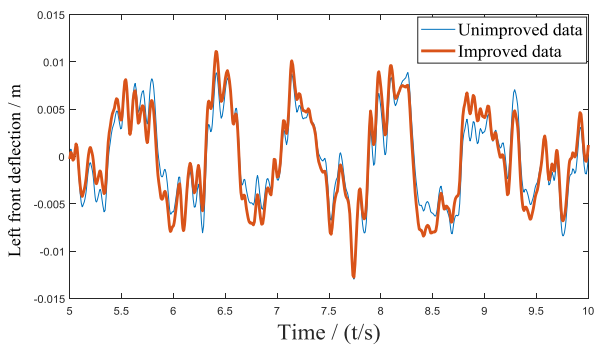

Figure 6. Comparison of left front dynamic deflection.

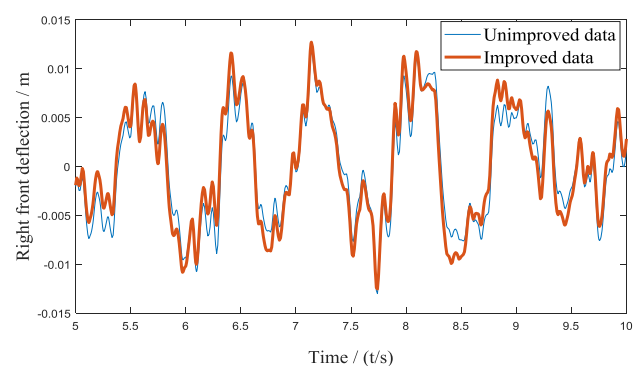

Figure 7. Comparison of right front dynamic deflection.

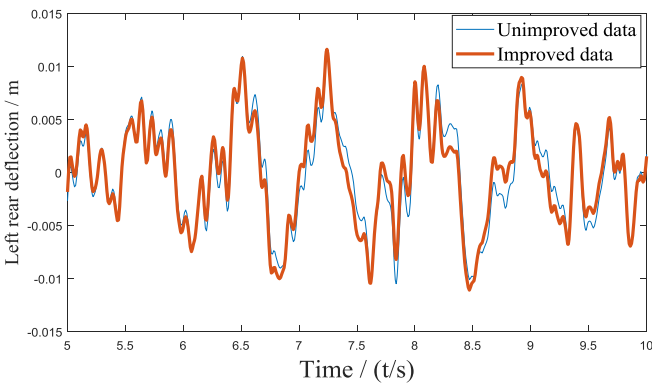

Figure 8. Comparison of left rear dynamic deflection.

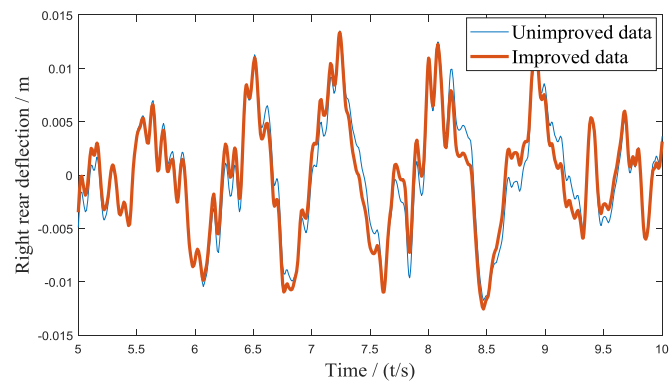

Figure 9. Comparison of right rear dynamic deflection. 
In order to perform more clearly, we calculate the root mean square values of the body accelerations, the RMS values of the driver seat accelerations and the RMS values of the dynamic deflections, as shown in the Table 2 .

Table 2. Comparison of performance indicators

\begin{tabular}{|c|c|c|c|}
\hline Stiffness & Body & Driver seat & Left front \\
$\mathbf{N} / \mathbf{m}$ & $\mathbf{a c c e l e r a t i o n}$ & acceleration & deflection \\
\hline $\boldsymbol{k}_{\mathbf{1}}=\boldsymbol{k}_{\mathbf{2}}=17$ & $\mathbf{m} \cdot \mathbf{s}^{-2}$ & $\mathbf{m m}$ \\
$\boldsymbol{k}_{\mathbf{3}}=\boldsymbol{k}_{\mathbf{4}}=22$ & 0.4150 & 0.6364 & 4.4 \\
\hline $\boldsymbol{k}_{\mathbf{1}}=\boldsymbol{k}_{\mathbf{2}}=13$ & & & \\
$\boldsymbol{k}_{\mathbf{3}}=\boldsymbol{k}_{\mathbf{4}}=18$ & 0.3899 & 0.5944 & 4.3 \\
\hline
\end{tabular}

\begin{tabular}{|c|c|c|c|}
\hline Stiffness & $\begin{array}{c}\text { Left rear } \\
\mathbf{N} / \mathbf{m}\end{array}$ & right front & Right rear \\
$\mathbf{m m}$ & $\mathbf{m m}$ & $\mathbf{m m}$ \\
\hline $\boldsymbol{k}_{\mathbf{1}}=\boldsymbol{k}_{\mathbf{2}}=17$ & 4.3 & 5.3 & 4.9 \\
$\boldsymbol{k}_{\mathbf{3}}=\boldsymbol{k}_{\mathbf{4}}=22$ & & & \\
\hline $\boldsymbol{k}_{\mathbf{1}}=\boldsymbol{k}_{\mathbf{2}}=13$ & 5.3 & 4.4 & 5.0 \\
$\boldsymbol{k}_{\mathbf{3}}=\boldsymbol{k}_{\mathbf{4}}=18$ & & & \\
\hline
\end{tabular}

From Table 2, it can be seen that, as far as possible without affecting the dynamic deflection of the suspension, the root mean square values of the body accelerations and the driver seat accelerations have been reduced and ride comfort of the vehicle have been improved.

\section{Conclusion}

The simulation design of the vehicle suspension system is carried out by using MATALAB / SIMULINK. The simulation curve of the vehicle suspension system is obtained, so that the vehicle parameter designer can predict the ride comfort of the vehicle at the design stage and adjust parameters so that the suspension parameters are optimized.

The seven-degree-of-freedom three-dimensional vibration model selected in this paper only considers the hysteresis effect of the input of the front and rear wheel surfaces, and does not take the difference in the input of the left and right wheel into account. Compared with the eleven degrees of freedom model, can not reflect the effect of the seat. The vehicle's mass, center of mass, moment of inertia, and vertical vibration of the seat system, as well as the rotation and body force, are changed in the case of manned or loaded vehicles.

\section{References}

1. J.C. Dixon, Suspension Geometry and Computation, (2009)

2. M. Mitschke, H. Wallentowitz, Dynamik der Kraftfahrzeuge, 378(2004)

3. W. Zhao, D. Zhao, Y.W. Qu, Mod. Manuf. Tech. Eqpt. (2015).

4. J. Peng, J. He, X.H. Li, Y.K. Chen, Y. Cong, J. Pla. Univ. Sci. Tech., 10, 77(2009)

5. Y. Zhang, J. Zhang, Mech. Syst. Signal Pr., 20, 363(2006)

6. J. Chen, W. Chen, H. Zhu, M. Zhu, Trans. Chin. Soc. Agr. Mach., 41, 11(2010)

7. X.P. Wang, W.B. Liu, J.J. Huang, Z.Y. LI, J. Shandong Jiaotong Univ., (2010).

8. J. Feng, Y. H. Chen, Adv. Mater. Res., 510, 250 (2012) 